\title{
Neuronal populations in primary motor cortex encode bimanual arm movements
}

\author{
O. Steinberg ${ }^{1}$, O. Donchin ${ }^{2}$, A. Gribova ${ }^{1}$, S. Cardoso de Oliveira ${ }^{3}$, H. Bergman ${ }^{1}$, E. Vaadia ${ }^{1}$ \\ ${ }^{1}$ Department of Physiology and the Interdisciplinary Center for Neural Computation, The Hebrew University, Hadassah Medical \\ School, PO Box 12272, Jerusalem 91120, Israel \\ ${ }^{2}$ Department of Biomedical Engineering, Johns Hopkins University, 720 Rutland Ave. 416 Traylor Bldg., Baltimore, MD 21205, \\ USA ${ }^{3}$ Institut fuer Arbeitsphysiologie an der Universitaet Dortmund, Ardeystr. 67, 44139 Dortmund, Germany
}

Keywords: bimanual coordination, electrophysiology, Macaca mulatta, population vector, single-cell recording

\begin{abstract}
Previous studies have shown that activity of neuronal populations in the primary motor cortex (MI), processed by the population vector method, faithfully predicts upcoming movements. In our previous studies we found that single neurons responded differently during movements of one arm vs. combined movements of the two arms. It was, therefore, not clear whether the population vector approach could produce reliable movement predictions also for bimanual movements. This study tests this question by comparing the predictive quality of population vectors for unimanual and bimanual arm movements. We designed a bimanual motor task that requires coordinated movements of the two arms, in which each arm may move in eight directions, and recorded single unit activity in the $\mathrm{Ml}$ of two rhesus (Macaca mulatta) monkeys during the performance of unimanual and bimanual arm movements. We analysed the activity of $212 \mathrm{Ml}$ cells from both hemispheres and found that, despite bimanual related activity, the directional tuning and preferred directions of most cells were preserved in unimanual and bimanual movements. We demonstrate that population vectors, constructed from the activity of MI cells, predict accurately the direction of movement both for unimanual and for bimanual movements even when the two arms move simultaneously in different directions.
\end{abstract}

\section{Introduction}

Natural voluntary movements require coordination among limbs, joints and muscles. A prevailing approach in experimental motor research was isolation of a single movement parameter, by studying simplified movements (for example - movements around a single joint). While this approach proved fruitful for studying the gross organization of motor cortex, it does not provide insight into the emergence of more complex movements.

Simultaneous movements of the two arms constitute a relatively simple example of complex movements and may serve to test whether and how the brain generates unique representations of complex movements from their constituent elements, as suggested by Leyton \& Sherrington, (1917) ' $\ldots$ the motor cortex may be regarded as a synthetic organ for compounding [...] movements [...] from fractional movements'.

Very little is known about cortical involvement in bimanual coordination. Studies by Tanji and coworkers suggested that, except for a small zone, the MI is not involved substantially in bilateral, distal movements (Tanji et al., 1988; Aizawa et al., 1990). Another study also failed to find bimanual specific activity in MI when more proximal movements were tested (Kazennikov et al., 1999). In contrast, two groups, using different tasks (Kermadi et al., 1998; Donchin et al., 1998) reported strong bimanual related effects for MI neurons. In the latter, we demonstrated that for many of MI cells, 'bimanual related activity' was relatively insensitive to small

Correspondence: Dr Eilon Vaadia, as above.

E-mail: eilon@hbf.huji.ac.il

Received 20 August 2001, revised 28 February 2002, accepted 6 March 2002 differences in muscular activity and arm kinematics between bimanual movements and unimanual movements and concluded that neural activity in the MI, as well as SMA, can reflect specialized cortical processing associated with bimanual arm movements (Donchin, 1998).

The finding that the activity of MI cells does not depend only on the limb's movement per se but also on the larger context (such as whether the arm moves together with the other arm) is consistent with other recent findings suggesting that the MI is not only responsible for muscle activation but may be involved in 'higher' aspects of motor control (Georgopoulos et al., 1989; Porro et al., 1996; Zhang et al., 1997; Carpenter et al., 1999; Kakei et al., 1999).

However, bimanual related activity in the MI seems inconsistent with the notion that single cells in the MI are characterized by symmetric directional tuning around a 'preferred direction' and, thus, that a population of MI cells can represent the direction of upcoming movements (Schwartz et al., 1988; Georgopoulos et al., 1986; Kalaska et al., 1989; Caminiti et al., 1990a; Caminiti et al., 1990b; Schwartz, 1993). If the preferred direction of a neuron is not consistent in different contexts, the whole concept of a 'population vector' (PV) may loose its validity. Additionally, one may ask whether the PV method is appropriate for representing multiple directions simultaneously, as is required for execution of bimanual movements. In this study we show that the preferred directions of single cells are relatively well preserved across different movement types, and thus, PV analysis is a valid tool for describing bimanual movements, including simultaneous representation of the directions of the two arms. Preliminary reports of this work have been published previously (Steinberg et al., 1998; Donchin et al., 1999). 


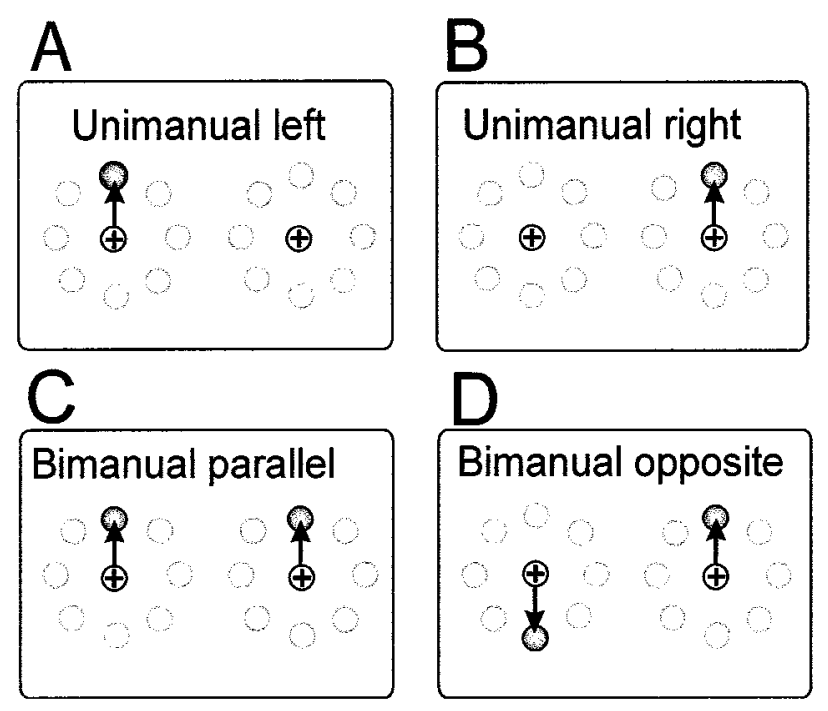

FIG. 1. The behavioural task with four types of movements shown in A-D The two cursors ('+' signs) showing the locations of the manipulanda, were placed here in the corresponding origins (centre circles). All possible target locations are shown as empty circles surrounding each origin (these are not visible to the monkey). The figure displays an example where the selected direction was $90^{\circ}$ (out of eight possible directions). The targets are marked as filled circles.

\section{Methods}

\section{Monkeys}

Two female rhesus (Macaca mulatta) monkeys, G and P (weighing 4-4.5 kg each), were used in the experiments. The monkeys were kept in the animal facilities of the faculty of medicine. The animals' care and surgical procedures used were in accordance with The NIH Guide for the Care \& Use of Laboratory Animals and the Hebrew University regulations. Unless mentioned specifically, all details of the behavioural task, surgical procedures, and recordings are identical to and can be found in Donchin et al., (1998).

\section{Behavioural task and training}

The two monkeys were trained to move two separate manipulanda, one with each arm. A trial began when the monkey aligned both cursors on 'origins' and held them still for $500 \mathrm{~ms}$. For each arm, one of eight peripheral target circles $(0.8 \mathrm{~cm}$ diameter $)$ could appear at a distance of $3 \mathrm{~cm}$ from the origin. The movement of each cursor was mapped to its corresponding manipulandum movement such that each millimeter of manipulandum movement caused one millimeter of movement of the cursor on the video display. The direction of movements from the origin to the right was defined as $0^{\circ}$, and movements away from the monkey (upward motion of the cursor) as $90^{\circ}$. There were four main types of trials (Fig. 1). In unimanual trials (Fig. 1A and B), only one target appeared and the monkey moved the appropriate arm and had to keep the other still. In bimanual trials (Fig. 1C and D), two targets appeared and the monkey moved each arm to the corresponding targets. As in Donchin et al. (1998), there were only two classes of bimanual movements that were tested: bimanual parallel (Fig. 1C, where the arms moved in the same direction) and bimanual opposite (Fig. 1D, where the directions of movement of the two arms differed by $180^{\circ}$ ). In contrast to Donchin et al. (1998), we recorded activity during performance of unimanual and bimanual movements in all eight directions, in all sessions.

\section{Surgery}

A head holder and two $27 \times 27 \mathrm{~mm}$ recording chambers were fixed onto the skull under general anaesthesia [induced by ketamine $(10-15 \mathrm{mg} / \mathrm{kg}$ ) and sustained with isoflurane] to allow recordings from the primary motor areas of both hemispheres. In Monkey $\mathrm{P}$, the implants were made of plastic to allow magnetic resonance imaging.

\section{Neural recordings}

Neural activity was recorded simultaneously by eight glass coated tungsten microelectrodes. Spike detection and online sorting was aided by $\mathrm{MSD}^{\circledR}$ (Alpha-Omega, Nazareth, Israel) spike sorters. All data, including spike shapes, were stored for off-line analysis.

\section{Experimental procedures}

Recording sessions started after full recovery from surgery (2-5 days later). In each session, two sets of four electrodes were inserted into the primary motor areas (MI) of the two hemispheres (one set into each hemisphere). The depth of each electrode was individually controlled and monitored by EPS ${ }^{\circledR}$ (Alpha-Omega). The recording area was selected on the basis of mapping sessions where we examined the effects of intracortical microstimulation (ICMS) and the neuronal activity evoked by passive manipulations of the monkeys' limbs. In addition, at the end of each recording session, we also tested ICMS effects and responses to passive limb manipulation from each of the eight electrodes. After completion of data collection, monkey $G$ was killed by injection of ketamine $(13 \mathrm{mg} / \mathrm{kg})$ followed by nembutal $(100 \mathrm{mg} / \mathrm{kg})$ and its brain was removed, dissected, and analysed histologically. Monkey $\mathrm{P}$ is still participating in experiments, and anatomical confirmation of the recording sites was only possible using MRI imaging.

\section{Data analysis}

\section{Tuning and preferred direction}

For most cells, the directional tuning curve can be approximated by a cosine function, although the method probably overestimates tuning width (Amirikian \& Georgopulos, 2000). We continued to use the cosine approximation in order to allow comparison of our results with the previous studies using population vectors to predict the direction of unimanual movements. The method we used to quantify the cells directional tuning and their 'preferred directions' (PD, the direction of movement to which the cell has the strongest response) was similar to the one used by Georgopoulos et al., (1982). We used the coefficient of determination, $R^{2}$, to quantify the fit of the neurons' directional tuning to a cosine function, and defined all cells with an $R^{2}$ above 0.7 as 'directionally tuned' and the others as 'nontuned'. This $R^{2}$ threshold was selected to facilitate comparison to previous PD studies (e.g., Georgopoulos et al., 1982; Schwartz, 1992).

We were interested in the degree of similarity of PDs in different types of movement, therefore, we compared PDs in ipsilateral unimanual, bimanual parallel and bimanual opposite movements to the PDs in contralateral unimanual movements. We examined whether the distributions of the differences in PDs were spaced equally using the Rao test (Mardia, 1972).

\section{Measuring directional tuning and PDs under different behavioural conditions}

A few possible methods could be used to quantify a single PD for each cell in different types of movement. In principle, we could have calculated four different preferred directions from each type of movement and calculate a simple average of the four measures to get 
one measure for each cell. However, such a method wouldn't have taken into account the magnitude of response in the different movement conditions. Therefore, we used the following technique to calculate a single estimate of a PD for each cell: we calculated five different PDs for each cell. The first four were taken from the neurons' activity during execution of the four different types of movement (unimanual right, unimanual left, bimanual parallel, and bimanual opposite). The fifth PD was computed by fitting one PD for each cell, based on its activity during performance of all types of movement. It was called the 'best-fit PD' (BFPD). To compute it, we used a least-squares fit that finds the best parameter set to fit the actual data recorded, with the restriction that the $\operatorname{PD}\left(\theta_{0}\right)$ is constant for all types of movement (constr function, optimization toolbox, MATLAB, Mathworks Inc.). The tuning function to which we fitted the data is thus:

$$
y_{T}(\theta)=a_{T}+c_{T}+\cos \left(\theta-\theta_{0}\right)
$$

where $y_{T}(\theta)$ is the firing rate of the cell in a certain type of movement $(T)$ in direction $\theta, a_{T}$ and $c_{T}$ are the parameters of the cosine function (specific for the movement type $T$ ) and $\theta_{0}$ is the preferred direction, which is assumed to be the same in all four types of movement. The movement direction $\theta$ in bimanual opposite trials was assigned according to the arm yielding stronger activation for that cell. This was determined according to the amplitude of the fitted cosine. For example, if a cell responded more strongly to unimanual movements of the left arm, then the movement direction was determined according to the direction of movement of the left arm regardless of the hemisphere the cell was recorded from. However, in some analyses (specified below), the movement direction in bimanual opposite trials was assigned according to the contralateral arm. $R^{2}$ was calculated for the fit of 32 data points to the four cosines.

In order to check that the tuning characteristics do not result from random variation in the firing rates, we tested whether the distribution of $R^{2}$ for the cosines around BFPDs, could be obtained by chance. Therefore, the distribution of the original $R^{2}$ values was compared to a distribution of $R^{2}$ values that were calculated by creating four cosines, based on the recorded data, with noise equal to the noise in the original data, but with random preferred directions. To do that we performed the following calculations: (i) the values of the cosine function fitted to the data were subtracted from the eight points of data, for each type of movement. (ii) These residuals were randomly ordered. (iii) A PD was randomly chosen, and a cosine function was calculated from this random PD and from the amplitude and offset of the original cosine. (iv) The randomly ordered residuals were added to this new cosine. This procedure was repeated for data from the four types of movement. Then, four cosine functions, with the same preferred direction in all four of them, were fitted to the new 32 points. An $R^{2}$ value was calculated to provide an estimate for the goodness of fit of all points (total 32) to the four cosine curves. The Kolmogorov-Smirnov two-sample test was used to determine whether the distributions of the real and randomized $R^{2}$ values could have been drawn from the same population.

\section{Bimanual related effect}

The definition of the 'bimanual related effect' is shown in equation 2, where 'bimanual' and 'unimanual' represent the cell's firing rates in a selected bimanual or unimanual movement, respectively.

$$
\text { Bimanual related effect }=\frac{\mid \text { bimanual }- \text { unimanual } \mid}{(\text { bimanual }+ \text { unimanual })}
$$

To select the movements for comparison, we first chose the direction of movement closest to the BFPD of the cell and the direction $180^{\circ}$ away from it. We compared each bimanual movement, in each of those directions (four comparisons), to the stronger response in the two unimanual movements composing it (as in Donchin et al., 1998). Then, we used the comparison that gave the largest difference to calculate the bimanual related effect. To evaluate the correlation between the bimanual related effect and the $R^{2}$ values we used the Spearman rank correlation coefficient.

\section{Population vectors}

Population vectors have been calculated in the customary fashion (Georgopoulos et al., 1986). In this study, we extracted three different types of PV values from the activity of the neurons: (i) for each movement type, the PV was constructed using the PD determined in that specific movement type. (ii) For all kinds of movements, the PD of contralateral unimanual movements was used, and (iii) a common PD was determined by the BFPD and used for all types of movements.

The error between the PV prediction and the actual movement direction was calculated as the mean of errors in all eight directions. A bootstrap analysis was used to estimate the confidence interval of differences in the average error for the different methods of calculating population vectors. In this analysis we calculated PV values using random weighting functions. Each such weighting function was a number randomly chosen from a distribution with a mean and standard deviation equal to those of the real weighting function. We repeated this 200 times, and each time we calculated the mean error of the direction of the resulting population vectors across the eight directions. This gave us a population of 200 averaged errors, from which we estimated the standard error of the mean.

\section{Calculating population vectors for simultaneous movements of two arms}

In order to arrive at two PV values, one for each arm, we divided the cells into two subpopulations using two approaches. First, we followed the classical hypothesis that each hemisphere controls the contralateral arm, and divided the cells by the hemisphere in which they resided. Second, cells were assigned to a subpopulation controlling the arm for which the modulation depth of the unimanual tuning-curve was larger (preferred arm, PA) regardless of its anatomical location. The population vectors were calculated in both cases using the BFPD. In calculating population vectors from subpopulations selected according to hemisphere, we used BFPDs in which the direction of bimanual opposite movements was assigned according to the contralateral arm. For population vectors based on subpopulations divided according to modulation depth in unimanual movement, we used BFPDs in which the direction of bimanual opposite movements was assigned according to the stronger activity in unimanual movements (the PA).

\section{Length of the population vectors}

The length of the population vector is always equal to or larger than zero. Small PV values may reflect a slow or small movement in a given direction, or they may be the outcome of 'random' directionless population activity. Therefore, it is important to estimate if a given vector's length is significantly different from one resulting from 
TABLE 1. The number and percentage of cells tuned to different types of movement

\begin{tabular}{lll}
\hline Movement type & Cells $(n)$ & Cells $(\%)$ \\
\hline Unimanual, ipsilateral & 54 & 34 \\
Unimanual, contralateral & 86 & 55 \\
Bimanual, parallel & 94 & 60 \\
Bimanual, opposite & 76 & 48 \\
\hline
\end{tabular}

The groups are not mutually exclusive. The total number of cells tuned for at least one movement type is 156 . The $R^{2}$ threshold for significance is 0.7 .
TABLE 2. The numbers and percentages of cells tuned in different combinations of types of movement.

\begin{tabular}{lcc}
\hline $\begin{array}{l}\text { Number of types } \\
\text { of movement }\end{array}$ & $\begin{array}{l}\text { Cells } \\
(n)\end{array}$ & $\begin{array}{l}\text { Cells } \\
(\%)\end{array}$ \\
\hline 0 (nontuned) & 56 & 26 \\
1 type & 64 & 30 \\
2 types & 44 & 21 \\
3 types & 34 & 16 \\
4 types & 14 & 7 \\
Total cells & 212 & 100 \\
\hline
\end{tabular}

Note that only $26 \%$ of the cells are not tuned to any type of movement. Fortyfour percent of all cells (which constitute 59\% of the 156 tuned cells) are tuned to more than one type of movement.
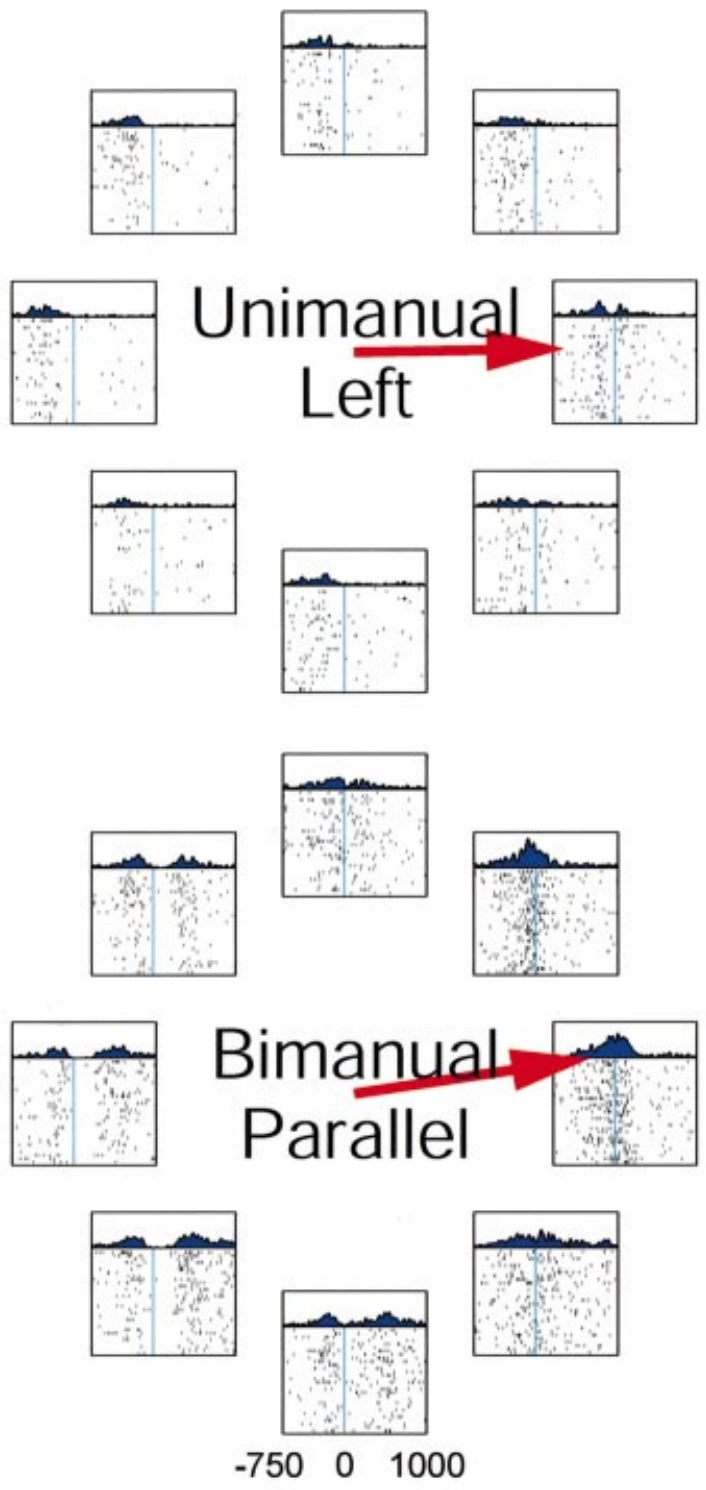

FIG. 2. An example of a cell with tuned responses in different types of movement. The figure depicts activity of one cell from the left MI during performance of four different types of movement. Each quadrant of the figure shows the activity of the cell in one type of movement, in eight directions. The rasters are aligned around movement onset (cyan line, time 0) in a time window of $750 \mathrm{~ms}$ before movement onset till $1000 \mathrm{~ms}$ after it. The red arrows indicate the preferred direction. Their lengths are proportional to the $R^{2}$ of the cosine fit. The cosine fit of this cell with its $R^{2}$ values and directional indices are shown in Fig. 4. 
random activity. To do this, we calculated the distribution of PV values that would have resulted from a population for which the weighting functions and the PDs are independent, and checked whether the real PV length was significantly different from the expected from this distribution.

\section{Results}

\section{Database}

Neural activity was recorded from the MI in 13 sessions in monkey $\mathrm{G}$, and 12 sessions in monkey P. Overall, we recorded the activity of 415 cells (207 in monkey $\mathrm{G}$ and 208 in monkey $\mathrm{P}$ ). The activity of each cell was evaluated from the recording period in which its firing rate was stationary. A number of additional criteria were used to include cells in the database: the anatomical location of the cell (see Methods), the quality of isolation, the number of successful trials performed during it's recording, and the total number of spikes fired. Two hundred and twelve cells fulfilled all these criteria (135 in monkey G, 66 in the left hemisphere and 69 in the right hemisphere, and 77 in monkey P, 37 in the left hemisphere and 40 in the right hemisphere).

\section{Directional tuning in unimanual and bimanual movements}

Cells are directionally tuned in different types of movement

As is expected from repeated reports of the arm area in the MI, most of the cells in our sample (156/212) exhibited broad symmetrical directional tuning around a preferred direction for at least one movement type. Table 1 shows the percentages of tuned cells in different types of movement, and indicates that there are groups of cells in the MI, which contain directional information not only about the contralateral movements, but also about the direction of bimanual movements and unimanual movements of the ipsilateral arm. Few cells ( $7 \%$ of the sample, Table 2 ) were tuned significantly to all four types of movement. An example is shown in Fig. 2. However, a significant portion of the cells was tuned to more than one type of movement (Table 2).

\section{A. Bimanual Parallel}

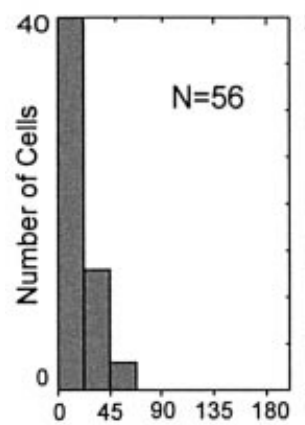

C. Ipsilateral

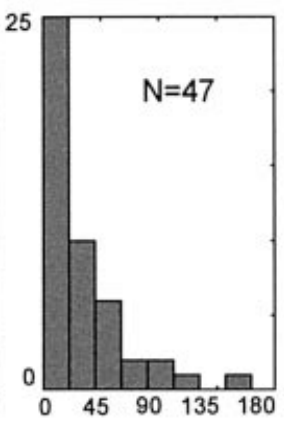

Difference (Degrees)

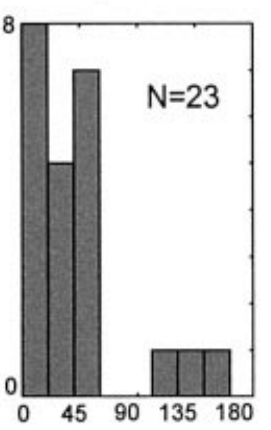

FIG. 3. Histograms of differences in PD values: (A) bimanual parallel vs. contralateral; (B) bimanual opposite vs. contralateral; (C) ipsilateral vs. contralateral. Only cells in which the $R^{2}$ was above 0.7 in both movement conditions being compared were included in the analyses. $\mathrm{N}$, number of cells included in each analysis.

\section{Preferred direction in different types of movements are similar}

The finding that a single neuron may be tuned to more than one type of movement calls for comparisons of PDs in different movement
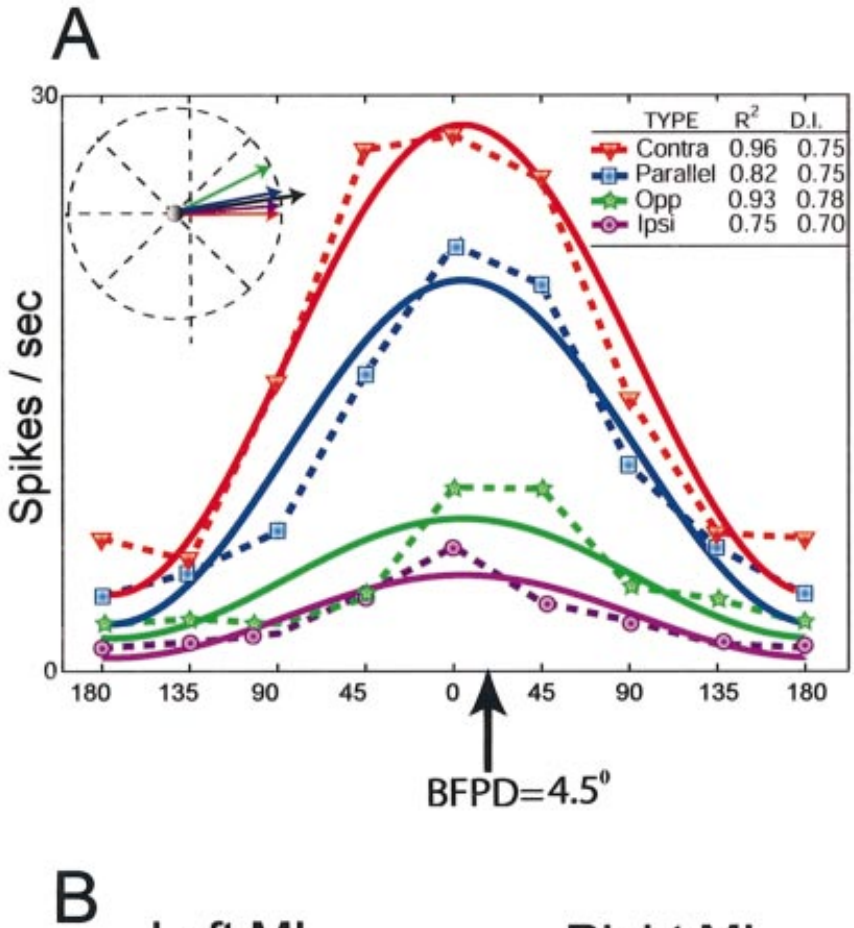

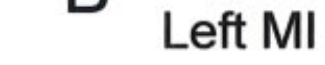

?ำ

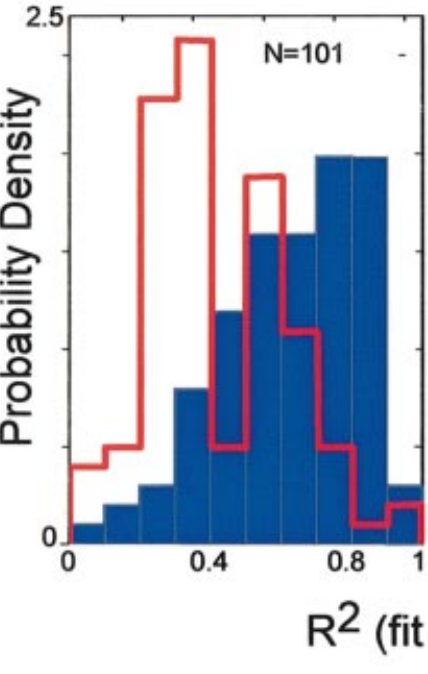

FIG. 4. (A) An example of best-fit preferred direction (BFPD) showing four cosines that fit to the activity of one cell (same cell as in Fig. 2) for four movement types, with the restriction that the preferred directions (peaks of all cosines) are the same. The BFPD for this cell was $4.5^{\circ}$ (marked by a vertical arrow below the horizontal-axis). The polar plot at the top-left corner shows the four preferred directions calculated separately for each type of movement (coloured arrows) and the BFPD (black arrow). For this particular cell all five measures are close to $0^{\circ}$, namely the cell is best tuned to arm movements to the right side. The $R^{2}$ values and directional index are also given in the figure. The directional index $(D I)$ is calculated as $D I=A / b_{0}$, where $b_{0}$ is the average firing rate across all movement types and $A$ is the cosine's amplitude, given by $A=\sqrt{b_{1}{ }^{2}+b_{2}{ }^{2}}$ (for details, see Georgopoulos et al., 1982). (B) $R^{2}$ value distribution validates BFPD. The figure shows histograms of $R^{2}$ values of the fit of BFPD for all cells (filled blue bars), and $R^{2}$ values calculated from randomly ordered data (red line). 
types. One possibility is that the cell's PD is fixed. If so, it could be represented either by the value measured during contralateral movements, or on the basis of the cell's activation during all types of movement. Another possibility is that the PD is not fixed and for each movement type the cell may 'prefer' a different direction. To begin studying these possibilities we tested whether the PDs measured during performance of different types are similar. The results are summarized in Fig. 3, where the distributions of differences of PDs (contralateral vs. bimanual parallel, contralateral vs. bimanual opposite, and contralateral vs. ipsilateral) are shown. In this analysis, we included only cells for which the $R^{2}$ values for the two compared types of movement were above 0.7 . All distributions were proven statistically to be nonuniform ( $P<0.01$, Rao test). The figure demonstrates that PDs in bimanual parallel movements had the greatest similarity to the PDs in the contralateral unimanual movements, while PDs in ipsilateral movements showed larger deviations.

We also tested the hypothesis that the PDs for the ipsilateral arm movement match the mirror-symmetric PD for movements of the contra-lateral arm. For example, if the PD for the contralateral arm is $0^{\circ}$, the expected matched PD for the ipsilateral arm is $180^{\circ}$, while for contralateral PD of $90^{\circ}$ the expected ipsilateral PD is $90^{\circ}$ as well. To perform that, we calculated the difference between the PD in ipsilateral movements and the 'mirror' of the PD of contralateral movements. The PD differences were much larger then those seen in Fig. 3C, therefore, we could not find support for the 'mirror' hypothesis in our data.

Can a cell be represented by one preferred direction?

As the PD of a single-cell in different types of movement were similar, but not identical, the next step was to estimate one PD for each cell based on its activity in different movement types. The algorithm for generating a unique PD (the BFPD) is described in the Methods section. Figure 4A shows the estimated BFPD of one cell. As the figure shows, this cell was tuned to all four types of movement, with quite similar PDs, and its BFPD was $4.5^{\circ}$ (black arrow). Note that the BFPD is indeed close to each of the four different PDs of this cell. Also note that the tuning curve for the contralateral arm (in red) seems to show better directionality tuning as compared to the tuning for the ipsilateral arm (in purple). However, computing a directionality index (see Georgopoulos et al., 1982) to each of the curves indicate that the modulation depth is similar in all conditions (for details see figure legend). This result is explained by the fact that the average firing rate across movements in all directions is highest for unimanual movements of the contralateral arm and lowest for movements of ipsilateral arm.

Figure 4B shows the distribution of $R^{2}$ values of the BFPDs for the whole population of cells (blue filled histogram) and the distribution of $R^{2}$ values calculated from the fit of a cosine model to randomized data (red line histogram). Comparing the two histograms shows that the distribution of $R^{2}$ of the cells is markedly shifted to the right (higher values) relative to the $R^{2}$ of bootstrapped data. The Kolmogorov-Smirnov two-sample test revealed that it is improbable that the distributions of the $R^{2}$ values calculated from the data and the distribution of the randomized $R^{2}$ values were drawn from the same population $(P<0.05)$. Half of the cells had BFPD $R^{2}$ values above $0.7(107 / 212)$.

\section{Bimanual related cells are also directionally tuned}

To examine the relation between bimanual related activity and directional tuning of MI cells, we first calculated the bimanual effect (see Methods). As expected from our previous work, we found that many cells exhibit a strong bimanual effect (in our sample, $52 \%$ of the cells shown in Fig. 5 had a bimanual related effect higher then $0.5)$. We then tested the relation between the strength of the bimanual related effect of each cell and it's cosine tuning, as estimated by the maximal $R^{2}$ value across the four types of movements. The results, as shown in Fig. 5, indicate that the correlation between the $R^{2}$ and the bimanual effect is very weak even if it is statistically significant (Spearman rank-based correlation coefficient, $r=-0.21, P<0.01$ ). The weak correlation suggests that many cells may encode both 'bimanuality' and movement direction independently.

\section{Population vector analysis}

In analysing population activity we first tested that the three conditions, which guarantee a good prediction of direction by population vectors (PV values), are met in our sample. The first - that the cells have symmetric tuning around the PD - was proven true by receiving high $R^{2}$ values (>0.7) for about $70 \%$ of the cells in at least one type of movement. The second - that the PDs of the cells we sampled were uniformly distributed in all directions - was tested by a Rao test. Here, we failed to show nonuniformity for $P>0.2$. The third is that the distributions of the amplitude and offset of the cosine functions are independent of the PDs. We ruled out correlation between those two parameters and the PDs (Spearman rank correlation coefficient, $r<0.1, P>0.3$ ).

Then, we calculated PV values for each direction and each movement type in three ways, generating three sets of PV values for each movement. First, for each type of movement, we used the preferred direction taken from the cells' activity during execution of that same type of movement. This generated the first set, called the four-PD set. This set must provide the most accurate prediction, as our data meets the conditions of Georgoupoulos et al. (1982). Indeed, the resulting PV values predicted movement directions quite accurately, in all movement types, as shown in Fig. 6A.

In the second and third set, the PV values were calculated using one preferred direction for each cell. As the classical approach for the motor cortex is that each hemisphere represents movements of the contralateral side of the body, we first chose the PD of the contralateral unimanual movements to generate a second set of PV values (the contralateral-based PV). As shown in Fig. 6B, these PV values were only accurate for the contralateral unimanual movements, and particularly inaccurate for predicting the movement of the ipsilateral arm.

Finally, we produced a third set of PV values, using the BFPD (BFPD-based PV values). The results of this analysis are shown in Fig. 6C. The first impression is that these PV values are almost as accurate as the best possible PV values (four-PDs set, compare Fig. 6C and A). To validate this impression, we calculated the average deviation of the different $\mathrm{PV}$ values from the actual direction of movement. Indeed, Fig. 7 shows that the prediction errors of BFPD-based PV values are not much larger than the four-PD based PV values. Thus, the results indicate that BFPDs represent the cells' tuning better than the preferred directions of the contralateral unimanual movements, and almost optimally.

\section{Population vectors represent simultaneous movements of the two arms}

To construct a separate population vector for each arm, we divided the population of sampled cells into two subpopulations, hypothesizing that bimanual movements are generated by two separate (although possibly coordinated) neuronal networks. The division into two subpopulations was performed in two different approaches. 


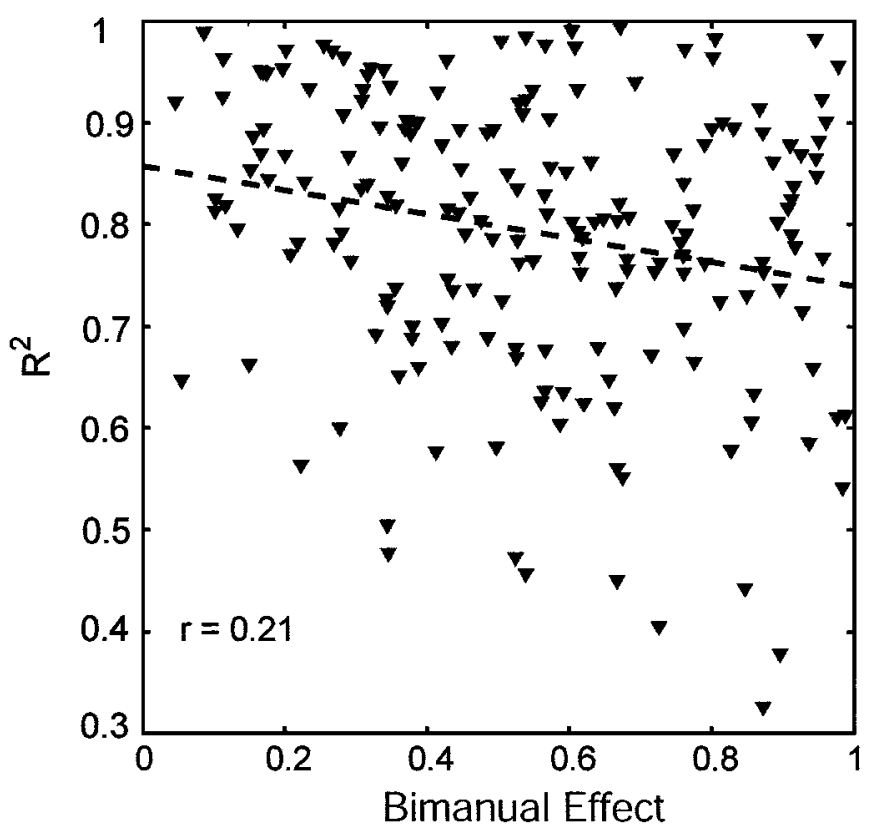

FIG. 5. $R^{2}$ values as a function of the strength of the bimanual related effect. Each triangle represents one cell. The Spearman rank correlation coefficient is -0.21 , indicating low negative correlation between the bimanual effect and the directional tuning of the cells. The dashed line shows linear regression fit.

The first natural choice was to divide the cells according to the hemisphere in which they resided. Figure 8A shows PV-pairs, for movements in $315^{\circ}$, each pair generated by the two subpopulations, one from the left hemisphere (for the right arm, in blue) and one from the right hemisphere (for the left arm, in red). The figure shows PV values for four types of movements. The two plots on the left show the prediction of unimanual movements. Note that for unimanual, movements, PV values were also obtained for the nonmoving arm. Although very small, these PV values did not point to random directions, but were generally aligned with the direction of the moving arm. PV values for bimanual movements are shown in the right side of the figure.

Figure $8 \mathrm{~B}$ shows predictions based of the second approach. Here, we selected cells for each arm on the basis of their activation, assuming that each cell can be characterized by its 'preferred arm' (PA), regardless of the hemisphere in which it resides. We assigned a PA for each cell on the basis of its activity in unimanual movements. Namely, the PA of a cell was the right arm if it was more active in unimanual right movements as compared to unimanual left movements. Table 3 shows the number of cells in the subpopulations selected by hemispheres and by the PA's of the cells, demonstrating that many cells have ipsilateral preference. Thus, the subpopulations differ by $28 \%$ of the cells. The result is in agreement with our previous report (Donchin et al., 1998). All 212 cells were included in the table because no inclusion criterion was used in the PV analysis. However, recreating the table using only cells that were tuned significantly to at least one type of unimanual movement did not substantially change the percentage of cells showing ipsilateral preference.

PV values for movements in the direction of $315^{\circ}$ generated by 'PA selection' of subpopulations are shown in Fig. 8B. Note that for this specific direction, the PA-based subpopulations represent the direction of simultaneous movements of the two arms somewhat better than selection by the hemispheric locations of the cells. Also note that the PV values for the nonmoving arm, are a little smaller in $\mathrm{B}$ as compared to A. For unimanual movements, this is an inevitable result of the reselection, but the improvement in the bimanual movements is not a trivial result. Yet, examining the PV values for all movement directions, we could not validate that the accuracy of PAbased PV values is higher then the hemisphere-based PV values.

\section{Discussion}

\section{Summary of results}

This paper investigates the activity of MI cells during performance of unimanual and bimanual arm movements, and demonstrates that directional tuning coexists with 'bimanual related activity' and that PDs of single cells in different types of movement are correlated. Further, we show that populations of cells can accurately predict the direction of movement even when the two arms move simultaneously in different directions.

\section{Directional tuning}

The previous finding of 'bimanual related activity' raised a simple question. Do single cells maintain directional tuning properties despite differences in activation intensity in unimanual and bimanual arm movements, or does the tuning change along with the activation intensity? The results of the current study show that the classical directional tuning properties that were described for unimanual movements (Georgopoulos et al., 1982) remain valid also for bimanual arm movements. Namely, firing rates of most cells are well described by a symmetric tuning curve around a preferred direction. Many cells showed directional tuning in more than one type of movement, and their preferred directions tended to remain similar in these different types. This finding is in agreement with a previous study where ipsilateral and contralateral responses were compared (Perepelkin \& Schwartz, 1996). For these cells, when a 'bimanual related effect' existed, it reflected significant changes of evoked activity not associated with a major change in the preferred direction of the cell. For example, Fig. 4A demonstrates a case where the 'bimanual related effect' reflected an overall change in firing rate without a shift of PD or change in the modulation depth.

\section{Cells code for more than one parameter of movement}

Our results are also in agreement with our own previous studies showing that many cells in the MI show a bimanual related effect (Donchin et al., 1998). Here, we extend these findings by showing that neuronal activities can depend both on the direction of movement and on the context of the arm movement, i.e., whether it is a unimanual movement or a bimanual arm movement in which the other arm is also moving (see Fig. 5). This also corroborates other studies showing that the activity of single-cells in the MI may be related to more than one parameter of the movement (Fu et al., 1993; Moran \& Schwartz, 1999).

We also looked at how these two parameters combine at the level of a single-cell. We demonstrated a way of describing the cell's activity as a function of a number of parameters of movement (see equation 1). The equation could be easily adapted into a general approach when more than one parameter of behaviour or stimulus may influence the cell's activity.

\section{Population vectors}

There is still debate over the physiological significance of the population vector. Some researchers argue that downstream struc- 


\section{A. Four Different PDs}

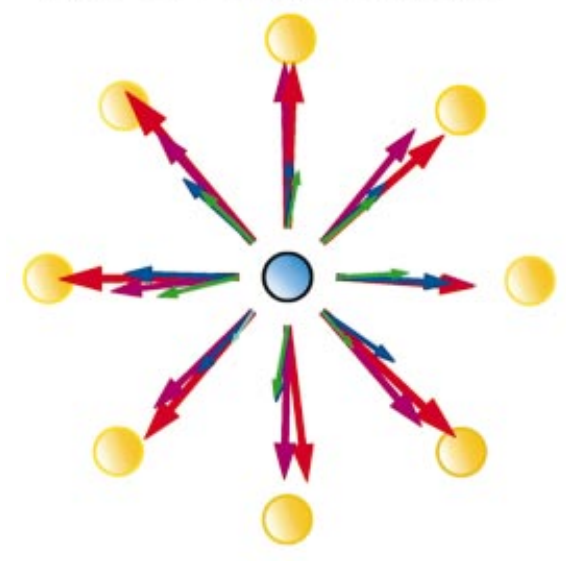

\section{B. PDs from Contralateral}

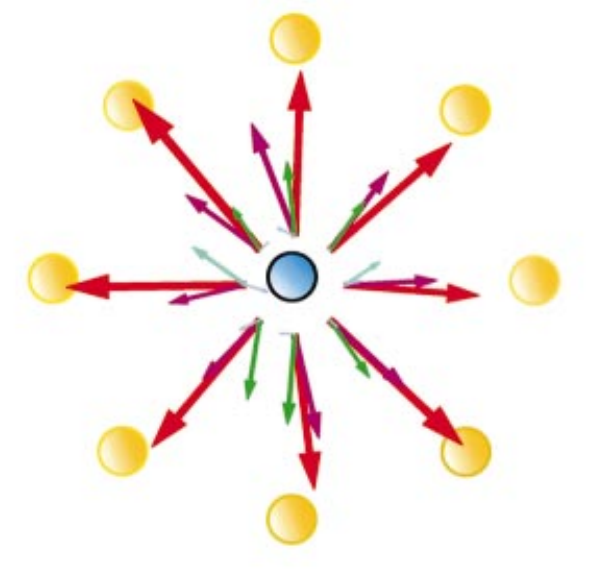

\section{PDs from BFPD}

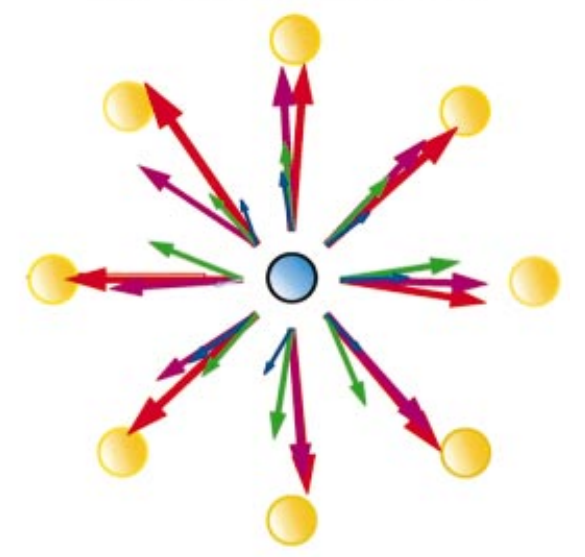

Significant

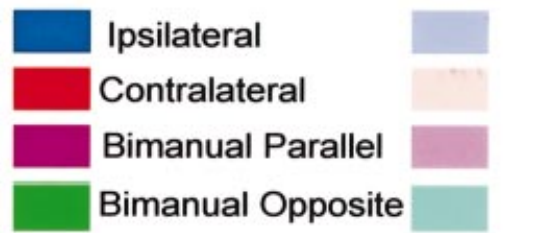

FIG. 6. Population vectors (PV values) calculated with different preferred directions. (A) PV values calculated using a different preferred direction for each type of movement. (B) PV values calculated using for each cell its preferred direction in contralateral movements. (C) PV values calculated using the BFPD, for all types of movement. The different colours represent the PV values of the different types of movements, as noted in the figure. Dark colours indicate PV values with lengths greater than the threshold of significance; Light colours indicate PV values with lengths smaller than the threshold of significance. Note, that the movement direction in bimanual opposite trials was assigned according to the preferred arm.

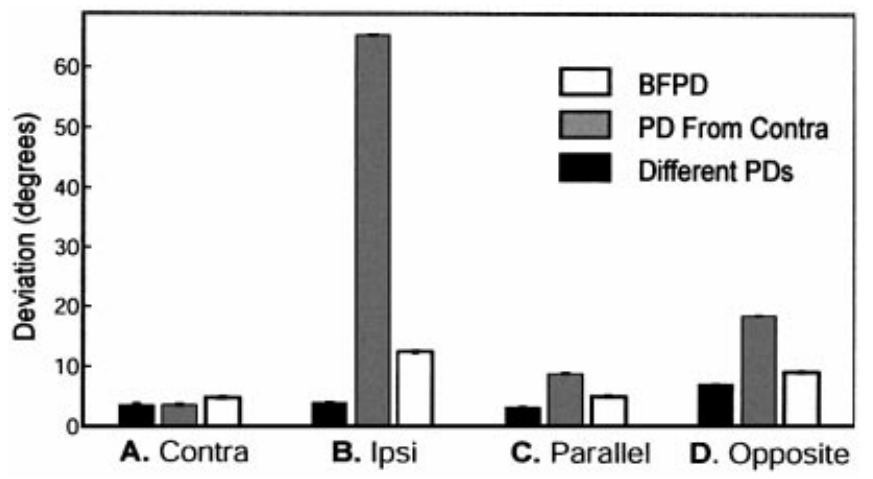

FIG. 7. Mean deviation of the population vectors (PV values) from the actual direction of movement. The deviations are shown for the three different methods of PV values calculation: (i) Four different preferred directions (black). (ii) The preferred direction of the contralateral movement (grey). (iii) The best-fit PD (BFPD - white). The error bars represent the standard error of the mean. Note that the BFPD method gives much smaller deviations in comparison to the method using the PDs from contralateral movements.

tures decode the signal from the MI by calculating a population vector. Others have argued that no real evidence exists that the PV is actually used to determine movement direction and that it is nothing more than a data-reduction technique. It has been further argued that the neurons giving rise to the PV may actually be muscle-related and not related to the arm kinematics (Mussa, 1988; Scott \& Kalaska,
1995; Todorov, 2000). Todorov, for example, presented a model, which '.. reinterprets the neural population vector to afford unified control of posture, movement and force production'.

Taken together, our previous accounts, that bimanual related activity was not sensitive to small variation of the movements of each arm or to muscular activity in bimanual vs. unimanual movements (Donchin, 1998), and the present result, that population activity codes for bimanual as well as unimanual movement direction, suggests that the motor cortex may also code for abstract movement parameters, independent of movement dynamics. This does not mean that motor cortex does not encode dynamic parameters of the movement. To conclude, while our experiment demonstrates that the MI contains information of abstract parameters of movements, it was not designed to differentiate between coding for intrinsic vs. extrinsic parameters of movements. It merely demonstrates that information about kinematics and context of the movement is present in motor cortex activity.

\section{Hemispheric control}

A more emphatic interpretation can be made on the issue of hemispheric control. The assumption that each hemisphere is related only to movements of the contralateral side of the body no longer seems accurate. There is ample evidence that the MI is active during ipsilateral movements (Evarts, 1966; Tanji et al., 1988; Kermadi et al., 1998). In addition, results obtained from human subjects using various techniques (Hoshiyama et al., 1997; Shibasaki, 1975; Kim et al., 1993; Chen et al., 1997) also demonstrate activation of the motor cortex during ipsilateral movements. Our findings are in 


\section{A. Hemispheric sub populations}

\section{Left Hemisphere \\ Right Hemisphere}

Unimanual Left

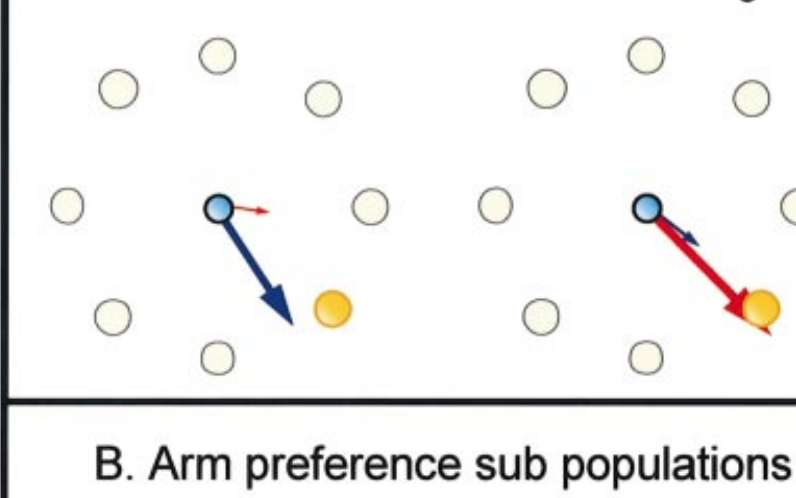

B. Arm preference sub populations
Bimanual Parallel

Bimanual Opposite
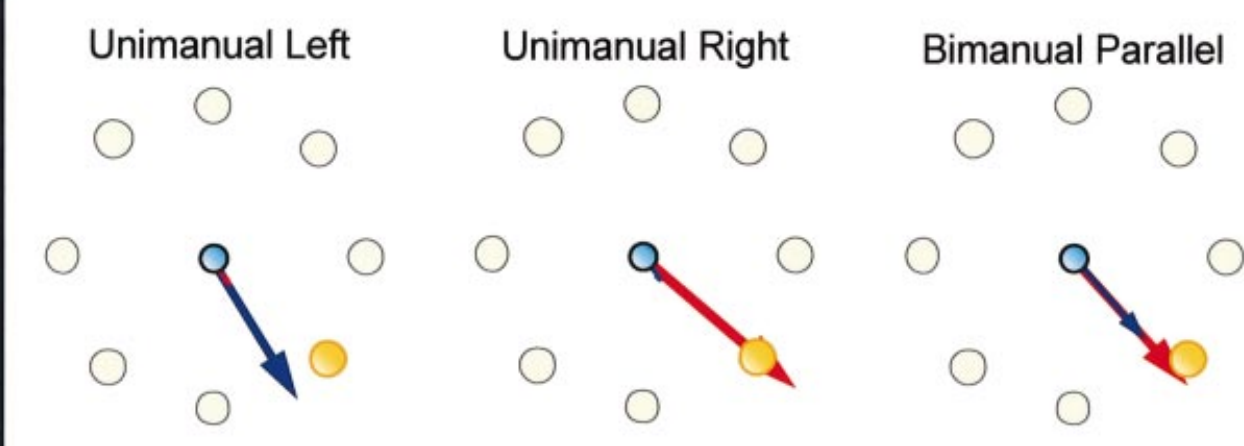

PA =Right

$\square \mathrm{PA}=\mathrm{Left}$

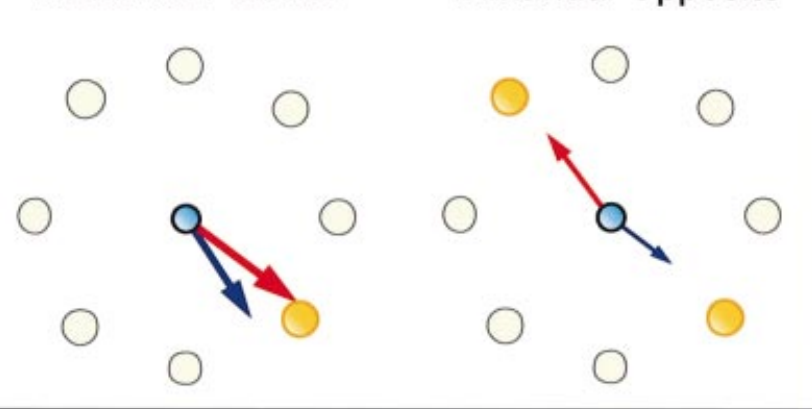

FIG. 8. Representation of simultaneous movements of the two arms to $315^{\circ}$ by pairs of PV values. (A) PV values constructed by dividing all cells into two subpopulations according to the hemisphere in which they reside. (B) PV values constructed by dividing all cells into two subpopulations according to their arm preference. The preferred direction used for PV calculation is the BFPD (but see text for description of the slightly different calculations in A and B).

TABLE 3. Ipsi- and contralateral preferences of right and left MIs

\begin{tabular}{ll}
\hline Side of hemisphere & Number of cells \\
\hline Left & \\
Preferred arm (Right) & 77 \\
Preferred arm (Left) & 24 \\
Right & \\
Preferred arm (Right) & 36 \\
Preferred arm (Left) & 75 \\
\hline
\end{tabular}

The table shows the size of subpopulations used to generate Fig. 8, according to hemisphere and preferred arm. The preferred arm was determined according to the amplitude of the cosine fit to the data (see Methods). Note, that overall, for $28 \%$ of the cells ( 24 cells from the left hemisphere and 36 cells from the right hemisphere) the ipsilateral arm was the preferred arm.

agreement with the notion that MI is active both during ipsilateral and contralateral movements.

Here we examined the 'natural' hypothesis that the two cerebral hemispheres collaborate in encoding the direction of movement of the two arms using two neuronal populations, each coding for one arm.
We divided the cells into two subpopulations either by hemisphere or by their arm preference. This type of division 'replaces' approximately a quarter of the cells in the contralateral hemisphere by cells from the ipsilateral one, and yet, the PV values calculated in bimanual movements from this division are not less accurate than PV values calculated when dividing by hemisphere. This result further supports the notion that both hemispheres are active and contribute to execution of both unimanual and bimanual movements. The way the two hemispheres interact and collaborate with each other, have been further examined, and is the subject of a separate study (Cardoso de Oliveira et al., 2001).

\section{Conclusions}

The results presented in this paper show that even though single cells have very different activities in unimanual and bimanual arm movements, they still maintain directional tuning. Thus, MI cells can represent both the direction of movement and its context. Reasonable population vectors are obtained under the assumption that the PDs of neurons in MI are independent of the movement type. The accuracies of PV values are equally good if populations are formed 
by hemisphere or by response, indicating that the cortical networks related to movements of both arms may be distributed over both hemispheres.

\section{Acknowledgements}

The research was supported in part by the Israel Science Foundation, founded by the Israel Academy of Sciences and Humanities, and by the German-Israel Binational Foundation (GIF). S. Cardoso de Oliveira is supported by the DFG (Ca 245/1-1). Y. Donchin provided help during the surgical procedures, G. Goelman provided help with the MRI and S. Haber with histological analysis. We also thank R. Paz for comments on an earlier version of the manuscript.

\section{Abbreviations}

BFPD, best-fit preferred direction; MI, motor cortex; PA, preferred arm; PD, preferred direction; $\mathrm{PV}$, population vector.

\section{References}

Aizawa, H., Mushiake, M., Inase, M. \& Tanji, J. (1990) An output zone of the monkey primary motor cortex specialized for bilateral hand movement. Exp. Brain Res., 82, 219-221.

Amirikian, B. \& Georgopulos, A.P. (2000) Directional tuning profiles of motor cortical cells. Neurosci. Res., 36, 73-79.

Caminiti, R., Johnson, P.B. \& Urbano, A. (1990a) Making arm movements within different parts of space: dynamic aspects in the primate motor cortex. J. Neurosci., 10, 2039-2058.

Caminiti, R., Johnson, P.B., Burnod, Y., Galli, C. \& Ferraina, S. (1990b) Shift of preferred directions of premotor cortical cells with arm movements performed across the workspace. Exp. Brain Res., 83, 228-232.

Cardoso de Oliveira, S., Gribova, A., Donchin, O., Bergman, H. \& Vaadia, E. (2001) Neural interactions between motor cortical hemispheres during bimanual and unimanual arm movements. Eur. J. Neurosci., 14, 1881-1896.

Carpenter, A.F., Georgopoulos, A.P. \& Pellizzer, G. (1999) Motor cortical encoding of serial order in a context-recall task. Science, 283, 1752-1757.

Chen, R., Cohen, L.G. \& Hallett, M. (1997) Role of the ipsilateral motor cortex in voluntary movement. Can. J. Neurol. Sci., 24, 284-291.

Donchin, O., Cardoso de Oliveira, S. \& Vaadia, E. (1999) Who tells one hand what the other is doing: the neurophysiology of bimanual movements. Neuron, 23, 15-18.

Donchin, O., Gribova, A., Steinberg, O., Bergman, H. \& Vaadia, E. (1998) Primary motor cortex is involved in bimanual coordination. Nature, 395, 274-278.

Evarts, E.V. (1966) Pyramidal tract activity associated with a conditioned hand movement in the monkey. J. Neurophysiol., 29, 1011-1027.

Fu, Q.G., Suarez, J.I. \& Ebner, T.J. (1993) Neuronal specification of direction and distance during reaching movements in the superior precentral premotor area and primary motor cortex of monkeys. J. Neurophysiol., 70, 20972116.

Georgopoulos, A.P., Kalaska, J.F., Caminiti, R. \& Massey, J.T. (1982) On the relations between the direction of two-dimensional arm movements and cell discharge in primate motor cortex. J. Neurosci., 2, 1527-1537.

Georgopoulos, A.P., Lurito, J.T., Petrides, M., Schwartz, A.B. \& Massey, J.T. (1989) Mental rotation of the neuronal population vector. Science, 243, 234-236.

Georgopoulos, A.P., Schwartz, A.B. \& Kettner, R.E. (1986) Neuronal population coding of movement direction. Science, 233, 1416-1419.

Hoshiyama, M., Kakigi, R., Berg, P., Koyama, S., Kitamura, Y., Shimojo, M.,
Watanabe, S. \& Nakamura, A. (1997) Identification of motor and sensory brain activities during unilateral finger movement: spatiotemporal source analysis of movement-associated magnetic fields. Exp. Brain Res., 115, 6 14.

Kakei, S., Hoffman, D.S. \& Strick, P.L. (1999) Muscle and movement representations in the primary motor cortex. Science, 285, 2136-2139.

Kalaska, J.F., Cohen, D.A., Hyde, M.L. \& Prud'homme, M. (1989) A comparison of movement direction-related versus load direction-related activity in primate motor cortex, using a two-dimensional reaching task. $J$. Neurosci., 9, 2080-2102.

Kazennikov, O., Hyland, B., Corboz, M., Babalian, A., Rouiller, E.M. \& Wiesendanger, M. (1999) Neural activity of supplementary and primary motor areas in monkeys and its relation to bimanual and unimanual movement sequences. Neuroscience, 89, 661-674.

Kermadi, I., Liu, Y., Tempini, A., Calciati, E. \& Rouiller, E.M. (1998) Neuronal activity in the primate supplementary motor area and the primary motor cortex in relation to spatio-temporal bimanual coordination. Somatosens. Mot. Res., 15, 287-308.

Kim, S.G., Ashe, J., Hendrich, K., Ellermann, J.M., Merkle, H., Ugurbil, K. \& Georgopoulos, A.P. (1993) Functional magnetic resonance imaging of motor cortex: hemispheric asymmetry and handedness. Science, 261, 615617

Leyton, A.S.F. \& Sherrington, C.S. (1917) Observations on the excitable cortex of the chimpanzee, orangutan, and gorrilla. Q. J. Exp. Psychol., 11, $135-222$.

Mardia, K.V. (1972) Statistics of Directional Data. Academic Press, London. Moran, D.W. \& Schwartz, A.B. (1999) Motor cortical representation of speed and direction during reaching. J. Neurophysiol., 82, 2676-2692.

Mussa Ivaldi, F.A. (1988) Do neurons in the motor cortex encode movement direction? An alternative hypothesis. Neurosci. Lett., 91, 106-111.

Perepelkin, P.D. \& Schwartz, A.B. (1996) Simultaneous populations of singlecell activity recorded bilaterally in primate motor cortex. Soc. Neurosci. Abstr., 22, 2022.

Porro, C.A., Francescato, M.P., Cettolo, V., Diamond, M.E., Baraldi, P., Zuiani, C., Bazzocchi, M. \& di-Prampero, P.E. (1996) Primary motor and sensory cortex activation during motor performance and motor imagery: a functional magnetic resonance imaging study. J. Neurosci, 16, 7688-7698.

Schwartz, A.B. (1992) Motor cortical activity during drawing movements: Single-unit activity during sinusoid tracing. J. Neurophysiol., 68, 528-541.

Schwartz, A.B. (1993) Motor cortical activity during drawing movements: population representation during sinusoid tracing. J. Neurophysiol., 70, 28 36.

Schwartz, A.B., Kettner, R.E. \& Georgopoulos, A.P. (1988) Primate Motor Cortex and Free Arm Movements to Visual Targets in Three-Dimensional Space: I. Relations between single cell discharge direction movement. $J$. Neurosci., 8, 2913-2927.

Scott, S.H. \& Kalaska, J.F. (1995) Changes in motor cortex activity during reaching movements with similar hand paths but different arm postures. $J$. Neurophysiol., 73, 2563-2567.

Shibasaki, H. (1975) Movement-associated cortical potentials with unilatera and bilateral simultaneous hand movement. J. Neurol., 209, 189-198.

Steinberg, O., Donchin, O., Gribova, A., Cardoso de Oliveira, S., Bergman, H. \& Vaadia, E. (1998) Can population vectors code bimanual movements? Neurosci. Lett. Suppl., 51, S40-S40.

Tanji, J., Okano, K. \& Sato, K.C. (1988) Neuronal activity in cortical motor areas related to ipsilateral, contralateral, and bilateral digit movements of the monkey. J. Neurophysiol., 60, 325-343.

Todorov, E. (2000) Direct cortical control of muscle activation in voluntary arm movements: a model. Nature Neurosci., 3, 391-398.

Zhang, J., Riehle, A., Requin, J. \& Kornblum, S. (1997) Dynamics of single neuron activity in monkey primary motor cortex related to sensorimotor transformation. J. Neurosci., 17, 2227-2246. 